\title{
Concept of a Decision-Based Pedestrian Model
}

\author{
Cornelia von Krüchten ${ }^{1}$, Andreas Schadschneider ${ }^{1,2}$ \\ ${ }^{1}$ Institut für Theoretische Physik, Universität zu Köln \\ Zülpicher Str. 77, 50937 Cologne, Germany \\ cvk@thp.uni-koeln.de; as@thp.uni-koeln.de \\ ${ }^{2}$ Institut für Physikdidaktik, Universität zu Köln \\ Gronewaldstr. 2, 50931 Cologne, Germany
}

\begin{abstract}
We develop a decision-based model for pedestrian dynamics which is an extension of the Stochastic Headway Distance Velocity (SHDV) model for single-file motion to two dimensions. The model is discrete in time, but continuous in space. It combines perception, anticipation and decision-making with the simplicity and stochasticity that are characteristic for cellular automaton models. The basic concept is discussed and preliminary results show that the model yield realistic trajectories and fundamental diagrams.
\end{abstract}

Keywords: Modelling, Perception, Decision-Based model, Distance-to-Collision, SHDV model

\section{Introduction}

Research on pedestrian dynamics and crowd movement is generally based on two aspects: first, empirical and experimental observations provide direct insights and allow for an unmediated investigation. However, since experiments cannot be performed for any arbitrary situation due to ethical, financial or practical reasons, modelling and simulation help to analyse a wide variety of scenarios and to understand the underlying general mechanisms of pedestrian dynamics.

Over the years, model development has given rise to many different modelling approaches that can be roughly classified considering their fundamental properties (see e.g. [1, 2]). For now, we will focus on microscopic models that describe the individual behaviour and interaction of particles that represent the pedestrians. This class again contains different approaches. The group of acceleration-based models includes social-force models that are usually continuous in space and time and deterministic, based on [3]. They consider intrinsic and extrinsic forces due to the environment and surrounding pedestrians that directly influence the motion. By contrast, rule- or decision-based models are mostly stochastic and discrete in space and time. Using this approach, often realized as cellular automaton models, the agents' movement is determined by certain rules that consider the local environment and current situation of the pedestrian (see e.g. $[4,5,6]$ ). Therefore, cellular automata (CA) models are rather simple, however, the spatial discretisation can lead to unwanted artefacts. A third group describes velocity-based models for which the velocity of the pedestrian is determined based on environment and interaction.

In this contribution, we want to present a modelling approach that combines several aspects of different model types. In Sec. 2, the general concept and idea of this approach is presented, whereas Sec. 3 mainly focuses on the concrete execution. Sec. 4 approaches first few simulation results.

\section{Model concept}

As a basis, this model combines continuous space with discrete time. Discrete time steps, as they are used in cellular automaton models, provide the opportunity for laying down simple rules that directly determine the pedestrians' behaviour. In CA models this simplicity is gained at the expanse of artefacts due to a lack of spatial resolution. Integrating continuous space as in forced-based models, allows arbitrary values for position and velocity. This approach of combining continuous space with discrete time steps is 
already used in some models, see e.g. [7-13]. However, since the potential of this approach has not been fully explored yet, this model is supposed to be a first step in this direction.

The basic concept of the model is shown in Fig. 1. In each time step, a pedestrian takes a decision about his or her velocity for the next step. In this model, speed and direction of motion are determined separately and rely on cognition, e.g. the so-called distance-to-collision which is again directly determined by the environment of the agent and his/her own goals.

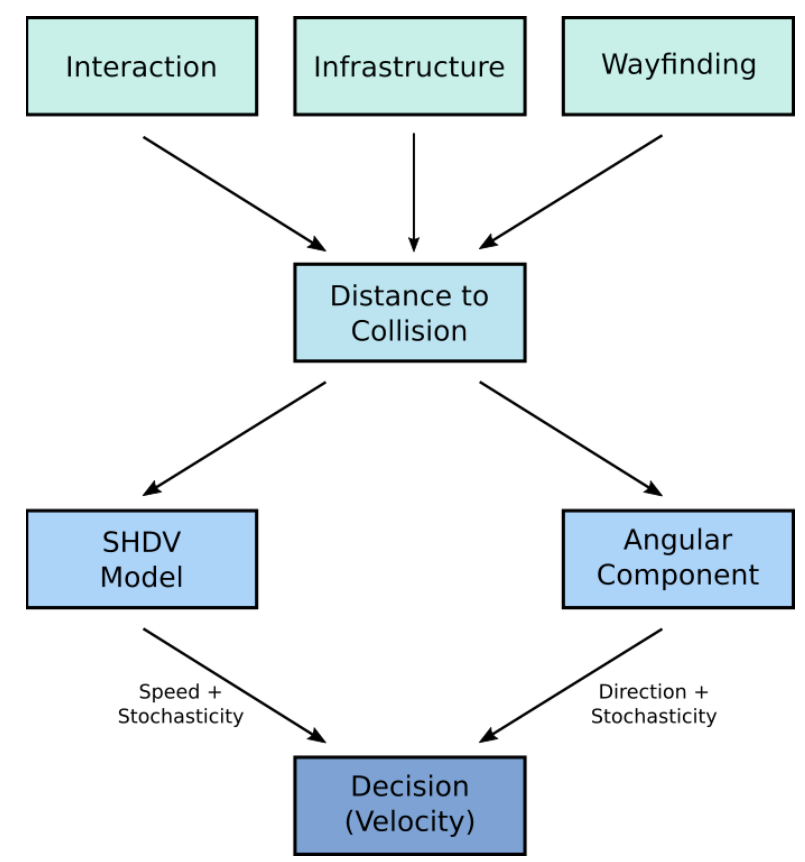

Fig. 1: Concept of the decision-based model: the environment including infrastructure, wayfinding and interaction determines the distance-to-collision which acts as a basis for the calculation of the velocity, namely speed and direction. These components include stochasticity to consider unknown mechanisms.

The environment of a pedestrian is perceived visually and consists of the infrastructure like walls, exits, etc., and other pedestrians the respective agent might interact with. Considering all these elements, the agent calculates the distance to a potential collision in a certain direction. Since anticipation was shown to be potentially crucial for pedestrian dynamics $[14,15]$, the distance-to-collision is not determined for this respective moment in time, but for the next time step, assuming uniform and linear movement of the agent itself as well as the other pedestrians. This quantity is then used as an input parameter for the calculation of speed and direction.

Since a pedestrian is assumed to prefer directions in which a collision with an infrastructure element or other pedestrians is at least unlikely, the distance-of-collision helps to weight the potential directions. Therefore, it is used to calculate a probability distribution for all directions. Moreover, the distance-to-collision also influences the speed of the pedestrian. In this case, it acts as an input parameter for the Stochastic Headway Dependent Velocity (SHDV) model [12] that correlates the distance headway of a pedestrian with his/her speed. It also includes a stochastic element that reduces the velocity of an agent additionally.

The stochasticity that is found in both speed and angular component of the velocity represents the limited knowledge about cognitive processes of pedestrians and shall cover underlying mechanisms that cannot be simulated or that are not fully understood. 


\section{Realization}

In contrast to many other approaches, we explicitly take into account the spatial extension of the pedestrians. They are modelled by a circle with radius $\mathrm{r}=0.15 \mathrm{~m}$ and are assumed to use cognitive abilities to move within the given environment. This behaviour includes several aspects that are explained separately in the following.

\subsection{Perception and visual field}

Visual perception is a commonly used approach in heuristic or perception-based models, see e.g. [13, 16-18]. In this model, each pedestrian perceives his or her environment via a visual field of range $d_{v f}$ and an expanse of $2 \varphi$. Only walls or pedestrians that lie within the visual field are perceived and considered for the calculation. In the visual field, pedestrians and walls or other obstacles cover a certain angular range that depends on the relative position and orientation to the perceiving agent (see Fig. 2). These ranges are then less preferable to be chosen for the direction of the velocity since the corresponding distance-to-collision is smaller than in angular ranges without any imminent obstacles. Considering all elements of the environment and all pedestrians results in an angular distribution of distances-to-collision that can be used to determine the direction for the next time step.

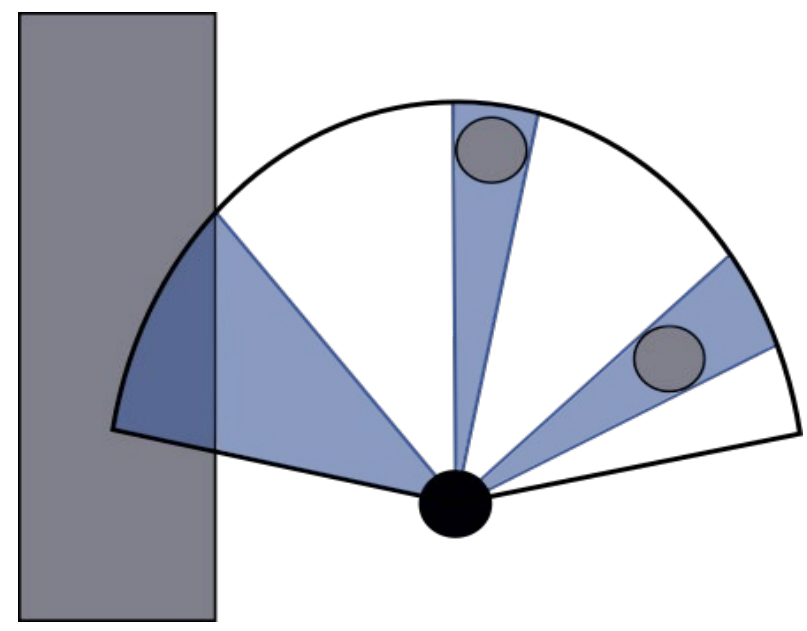

Fig. 2: Each pedestrian (black circle) has a visual field that can be partially covered by walls (left) or other pedestrians (grey circles). Directions chosen within these ranges are less likely.

\subsection{Anticipation and distance-to-collision}

In several model approaches, anticipation concepts are realised using the time or the distance to an imminent collision, see e.g. [8, 17, 19-21]. In this model, the anticipated distance-to-collision is calculated for each pedestrian that is perceived in the visual field of the agent. The agent assumed the he/she itself as well as the other pedestrian will move with the same speed and direction as in the previous step, leading to a uniform, linear motion. Then the distance-to-collision is calculated based on these anticipated positions with consideration of the extension of the bodies. Relying on anticipation shall help to consider the current direction of motion when taking the decision on the direction for the next step.

\subsection{Determination of direction of motion}

The determination of the angular component of the velocity is based on different aspects. First, each pedestrian is assigned a target position he or she wants to reach during the simulation. Therefore, for each time step the direction $\alpha_{\mathrm{t}}$ towards the target is determined. The final target angle is then calculated with a Gaussian distribution with mean $\alpha_{\mathrm{t}}$ and standard deviation $\mathrm{s}=0.05$. Using this stochasticity includes that a pedestrian does not always choose the perfect angle to make the simulation results more realistic. At this point the pedestrian considers the presence of walls etc. If the target direction eventually resulted in a 
collision with the infrastructure, the pedestrian would choose an intermediate target for his/her current calculation that is given by the surrounding walls and obstacles. How exactly this intermediate target is situated could be determined in more general settings by using different route choice or wayfinding procedures.

In the presence of other pedestrians, the preferred direction must be changed. During the perception phase the relative distances and orientations of all pedestrians to the respective agents are determined. This leads to an angular distribution of distances-to-collision. For angular ranges that are not covered by any pedestrians, the distance-to-collision is set to the maximum visual range $d_{\mathrm{vf}}$. When divided by this maximum range, each angle within the visual field is assigned a positive value for the distance-to-collision within 0 and 1 . This quantity can then be used as a measure of probability that the respective direction is chosen for the next time steps. Therefore, if a pedestrian $\mathrm{i}$ is perceived by an agent in the direction $\alpha_{i}$ and a respective distance-to-collision $d_{i}$, the probability for the agent choosing this direction for the next timestep is given by $\mathrm{p}_{\mathrm{i}}=\mathrm{d}_{\mathrm{i}} / \mathrm{d}_{\mathrm{vf}}$. Summing over all pedestrians results in an angular probability distribution $\mathrm{P}(\alpha)$ that is positive and stepwise constant. Then, according to this distribution, the direction is calculated that can be understood as an "interaction angle" (in order to distinguish it from the target angle).

Due to the stochastic nature of the collision avoidance procedure, large changes of direction could occur from one time step to another. To avoid this, a concept of inertia in case of interaction is applied. If the environmental situation has not changed compared to the previous time step, the pedestrian should keep the direction of rotation for the next time step. That means, if a pedestrian has chosen to turn left to avoid a collision in the previous time step, and there were no pedestrians exiting or entering the visual field, he or she should turn left again, i.e. chose any arbitrary direction on his left side. Only if the situation has changed during the last time step, there is no such restriction.

If an interaction angle is chosen, the distance-to-collision in this direction is again determined considering both environment and pedestrians. This distance is then compared to the distance-to-collision (considering infrastructure and pedestrians) in the direction to the target. Then, out of these two directions the one with the highest distance-to-collision is chosen to be the direction of velocity.

\subsection{Determination of speed}

The distance-to-collision resulting from the determination of the direction acts now as the input parameter for the determination of the absolute value of the velocity. Here, one relies on the SHDV model [12] that was originally developed for single-file motion. In this model, the velocity of an agent depends linearly on his/her headway distance $\mathrm{h}$ in one dimension which is replaced by the distance-to-collision in the two-dimensional case (see also Fig. 3):

$$
v(h)=\left\{\begin{array}{cl}
0 & h \leq d \\
\alpha(h-d)+v_{\min } & d<h<d_{c} \\
v_{\max } & d_{c} \leq h
\end{array}\right.
$$

with $\mathrm{v}_{\max }$ and $\mathrm{v}_{\min }$ representing the maximum and minimum velocity, respectively, $\mathrm{d}$ being a lower threshold and $d_{c}$ being a higher threshold resulting from $d_{c}=d+1 / \alpha\left(v_{\max }-v_{\min }\right)$. For a headway distance below $d$, a pedestrian stands still, with a headway distance that is larger as $d_{c}$ he or she moves freely with maximum velocity. $\alpha$ is the slope of the increasing headway-velocity function.

As a stochastic element, a slow-to-start rule [22] is additionally introduced. Pedestrians whose speed was zero in the last timestep stand still for the next time step with a probability $\mathrm{p}_{0}$, with probability $1-\mathrm{p}_{0}$ they move with a speed according to (1). 


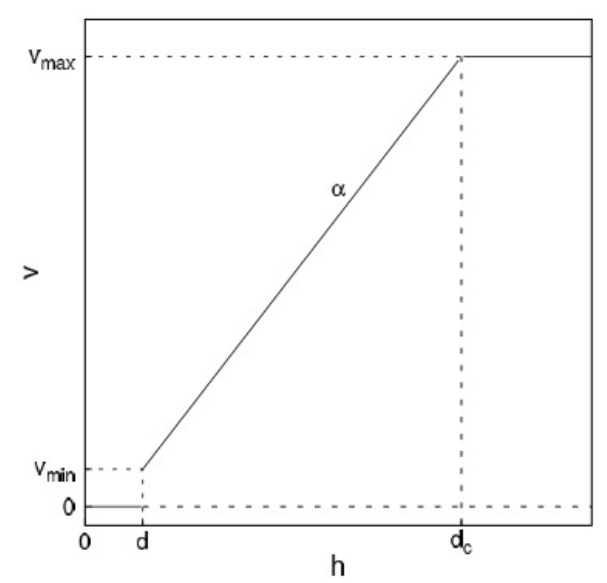

Fig. 3: Headway-velocity function of the SHDV model. The velocity increases linearly with the headway distance without exceeding the free-flow velocity $v_{\max }$ and being 0 for distances that are smaller than the lower threshold $\mathrm{d}$ (from [12]).

\section{First preliminary results}

To assess if the approach described above can reproduce realistic pedestrian dynamics, the model is tested with some simple scenarios. First, we simulated two-person-interactions without additional influence by the infrastructure; second, we measured a fundamental diagram for pedestrian motion in a corridor.

All simulations are performed with a time step of $\Delta t=0.3 \mathrm{~s}$ and parallel update. The maximum range of the visual field is set to $\mathrm{d}_{\mathrm{vf}}=8.0 \mathrm{~m}$. The parameters for the determination of speed are chosen as $\mathrm{v}_{\max }=$ $1.2 \mathrm{~m} / \mathrm{s}, \mathrm{v}_{\min }=0.1 \mathrm{~m} / \mathrm{s}, \alpha=0.5$ and $\mathrm{p}_{0}=0.5$, as in [12]. Compared against the original SHDV model, the lower threshold $\mathrm{d}$ was reduced to $0.15 \mathrm{~m}$ to compensate the explicit consideration of the pedestrians' extension.

The scenarios are not meant to be used for calibration or optimization. To approach the characteristics of the modelled dynamics we used reasonable values for the model parameters such as radius and lower threshold.

\subsection{Two-person-interaction}

Fig. 4 shows trajectories of simple scenarios with two pedestrians that pass in a free area. All situations were simulated with a visual field extension of $2 \varphi=170^{\circ}$.

In the first scenario, a pedestrian walking from the bottom to the top passes another pedestrian that stands in the middle of the room. As soon as the walking agent perceives the presence of the other pedestrian, it starts to avoid the collision. The trajectory during the avoidance phase is not as smooth as it is wanted to be. This indicates that the applied concept of inertia is not strong enough to suppress larger deviations of the turning angle. Moreover, since the trajectories in Fig. 4 are specific realizations of a stochastic process without any averaging, the simulations do not result in smoothed trajectories.

In Fig. 4 (b), two pedestrians pass on their way to the opposite site of the room. Since the pedestrian coming from the top (dashed green line) turns early enough, the other pedestrian can walk nearly unaffected through the room.

The last Fig. 4 (c) shows two pedestrians coming from the lower edges walking towards the opposite edges. As in (b), one pedestrian can nearly maintain the optimal route, because the other agent gives way. The temporal dimension cannot be shown in this depiction, therefore we would like to emphasise that the agents do not collide, but pass the position where the trajectories cross at different times. 


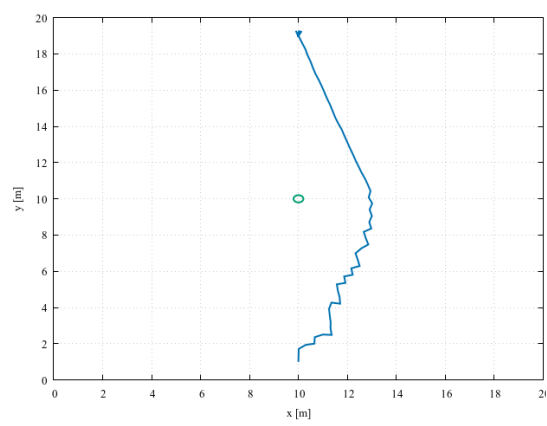

(a)

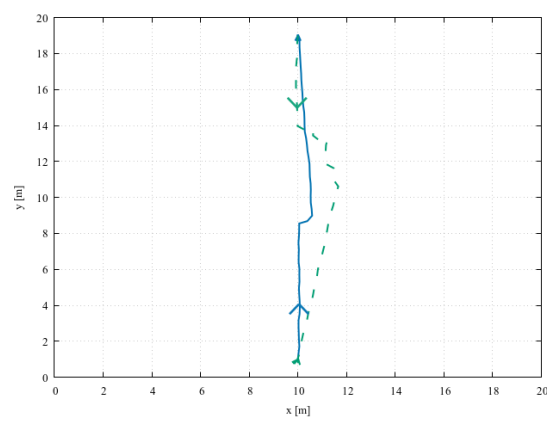

(b)

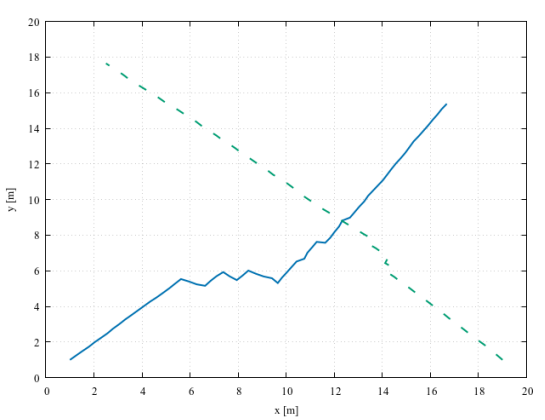

(c)

Fig. 4: Simple two-person-interaction scenarios: (a) a pedestrian walks bottom up with passing a standing pedestrian, (b) two pedestrians walking bottom up (blue solid line) / top down (green dashed line, the arrows show the respective direction of motion), (c) two pedestrian walking diagonally bottom down with left to right/vice versa. Since time is not depicted in this plot, it should be emphasised that the pedestrians do not collide in (c).

\subsection{Fundamental diagram of single file motion}

As a second test, we simulated the situation the SHDV model was originally developed for. The pedestrians walked in single-file motion, that means without any overtaking, in a corridor of length $\mathrm{L}$ $=26.0 \mathrm{~m}$ and width $\mathrm{B}=0.8 \mathrm{~m}$ with periodic boundary conditions. The density was measured globally by the number of agents in the corridor. Because of the extension of the pedestrians the maximum number of agents was by comparison to the SHDV model decreased from 70 to 60 . The simulation started with inhomogeneous initial conditions, meaning that all pedestrians were placed at the beginning of the corridor with a minimal distance of $3 \mathrm{~cm}$. Since backward motion is forbidden in single-file motion, the pedestrians were forced to walk towards their target when the interaction angle exceeds $0.9 \times \pi / 2$. Additionally, the range in which other pedestrians can be perceived was set to $2 \varphi=1.25 \times \pi$. All simulations were performed for $600 \mathrm{~s}$, measuring over the last $100 \mathrm{~s}$.

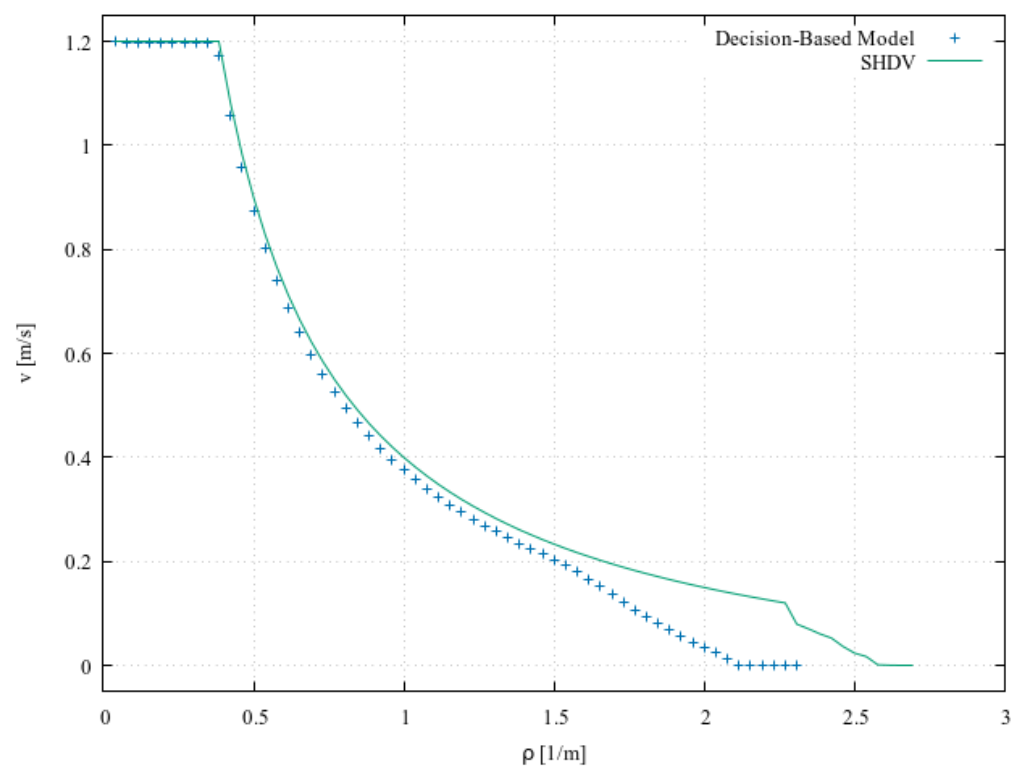

Fig. 5: Comparison of fundamental diagrams for single-file motion of the SHDV model and the decision-based approach. $\rho$ is the global density, and $v$ the averaged velocity. In general, the velocities in the decision-based model are lower than these of the SHDV model, the deviation increases with density. 
The fundamental diagram in Fig. 5 shows the global density against the averaged velocity. The progress of the curve fits qualitatively to the fundamental diagram of the SHDV model. However, the velocities of the decision-based model are lower than the velocities measured with the SHDV model. The origin for these deviations, especially for large densities, lies probably in the consideration of a finite extension of pedestrians. In the SHDV model, pedestrians were treated as point-like particles and an effective extension was introduced by the headway-velocity function.

\section{Conclusion}

The new modelling approach presented in this contribution is based on the combination of continuous space and discrete time and relies on cognition and perception. It is an extension of the one-dimensional SHDV model to two dimensions by adding an angular component to the pedestrians' velocity.

In each time step, a pedestrian perceives its environment visually and determines the distance-to-collision for different directions. In doing so, the agents consider the infrastructure as well as other pedestrians. Based on this information the pedestrians take a decision about their velocity for the next time step. The direction of motion is determined by the pedestrians' individual target and their interaction strategy. In order to avoid collisions, a probability distribution for all potential directions is calculated based on the distance-to-collision. Therefore, directions that may lead to a collision are less likely to be chosen for the next time step. Once a direction is chosen, the distance-to-collision in this direction is used as an input parameter for the dynamics of the SHDV model. So, the speed for the next time steps depends linearly on the distance-to-collision.

Using stochastic elements in the determination of direction and speed covers unknown underlying mechanisms and shall make the simulation results more realistic.

The model was tested with simple scenarios. In the two-person-interaction, it is shown that the model provides realistic trajectories, even if the inertia component of the collision avoidance must be improved. Comparing the fundamental diagram for single-file motion to the results of the SHDV model, it is shown that the model yields similar results. However, due to the extension of the pedestrians that is explicitly considered in this model, the averaged velocities are lower than in the SHDV model. This effect becomes even more significant for large densities. So far, the model has not been calibrated with empirical data. For the preliminary investigations reasonable parameter values were used without any optimization procedure.

\section{Acknowledgements}

Financial support by Deutsche Forschungsgemeinschaft (DFG) under grant SCHA 636/9-1 and Bonn-Cologne Graduate School of Physics and Astronomy (BCGS) is gratefully acknowledged.

\section{References}

[1] A. Schadschneider, W. Klingsch, H. Klüpfel et al., "Evacuation dynamics: empirical results, modelling and applications," in Encyclopedia of Complexity and Systems Science, R.A. Meyers, Ed. New York: Springer, 2009, pp. 3142-3176.

[2] A. Schadschneider, M. Chraibi, A. Seyfried et al., „Pedestrian dynamics - from empirical results to modelling", to appear in Crowd Dynamics, Volume 1 - Theory, Models, and Safety Problems, L. Gibelli and N. Bellomo, Ed. Springer.

[3] D. Helbing and P. Molnár, "Social force model for pedestrian dynamics," Phys. Rev. E, vol. 51, pp. 4282-4286, 1995.

[4] M. Fukui and Y. Ishibashi, "Jamming transition in cellular automaton models for pedestrians," $J$. Phys. Soc. Japan, vol. 68, pp. 3738-3739, 1999.

[5] V. Blue and J. Adler, "Cellular automata microsimulation of bidirectional pedestrian flow," Transp. Res. Rec., vol. 1678, pp. 135-141, 2000.

[6] C. Burstedde, K. Klauck, A. Schadschneider and J. Zittartz, „Simulation of pedestrian dynamics using a two-dimensional cellular automaton," Phys. A, vol. 295, pp. 507-525, 2001. 
[7] K. Yamamoto, S. Kokubo and K. Nishinari, „Simulation for pedestrian dynamics by real-coded cellular automata (RCA)," Phys. A, vol. 379, pp. 645-660, 2001.

[8] I. Karamouzas and M. Overmars, „A velocity-based approach for simulating human collision avoidance," in Intelligent Virtual Agents, Springer, 2010, pp. 180-186.

[9] G. Baglietto and D.R. Parisi, „Continuous-space automaton model for pedestrian dynamics,” Phys. Rev. E, vol. 83, no. 056117, 2011.

[10] Z.-M. Fang, W. Song, X. Liu et al., „A continuous distance model (CDM) for single-file pedestrian movement considering step-frequency and length," Phys. A, vol. 391, pp. 307-316, 2012.

[11] M.J. Seitz and G. Köster, „Natural discretization of pedestrian movement in continuous space,” Phys. Rev. E, vol. 86, no. 046108, 2012.

[12] C. Eilhardt and A. Schadschneider, „Stochastic headway dependent velocity model for 1d pedestrian dynamics at high densities," Transp. Res. Proc., vol. 2, pp. 400-405, 2014.

[13] W. Kang and Y. Han, „A simple and realistic pedestrian model for crowd simulation and application," arXiv:1708.03080, 2017.

[14] S. Nowak and A. Schadschneider, "Quantitative analysis of pedestrian counterflow in a cellular automaton model," Phys. Rev. E, vol. 85, no. 066128, 2012.

[15] Y. Suma, D. Yanagisawa and K. Nishinari, "Anticipation effect in pedestrian dynamics: modelling and experiments," Phys. A, vol. 391, pp. 248-263, 2012.

[16] G. Antonini, M. Bierlaire and M. Weber, "Discrete choice models of pedestrian walking behavior," Transp. Res. B, vol. 40, pp. 667-687, 2006.

[17] M. Moussaïd, D. Helbing and G. Theraulaz, "How simple rules determine pedestrian behavior and crowd disasters," PNAS, vol. 108, pp. 6884-6888, 2011.

[18] M. Zhou, H. Dong, F.-Y. Wang et al., "Modeling and simulation of pedestrian dynamics behavior based on a fuzzy logic approach", Information Sciences, vol. 360, pp. 112-130, 2016.

[19] A. Johansson, "Constant-net-time headway as a key mechanism behind pedestrian flow," Phys. Rev. E, vol. 80, no. 026120, 2009.

[20] P. Degond, C. Appert-Rolland, M. Moussaïd et al., "A hierarchy of heuristic-based models of crowd dynamics," J. Stat. Phys., vol. 152, pp. 1033-068, 2013.

[21] M. Asano and T. Iryo and M. Kuwahara, "Microscopic pedestrian simulation model combined with a tactical model for route choice behaviour," Transp. Res. C, vol. 18, pp. 842-855, 2010.

[22] R. Barlovic, L. Santen, A. Schadschneider and M. Schreckenberg, "Metastable states in cellular automata for traffic flow, “ Europ. Phys. J. B, vol. 5, pp. 793-800, 1998. 